\title{
Effects of let-7c on the proliferation of ovarian carcinoma cells by targeted regulation of CDC25a gene expression
}

\author{
WEI ZHANG ${ }^{1}$, QINGRU ZENG ${ }^{2}$, ZHENYING BAN ${ }^{1}$, JING CAO ${ }^{1}$, TIANJIAO CHU ${ }^{1}$, \\ DONGMEI LEI ${ }^{1}, \mathrm{CHI} \mathrm{LIU}^{3}$, WENTAO GUO ${ }^{4}$ and XIANXU ZENG ${ }^{1}$
}

\begin{abstract}
Departments of ${ }^{1}$ Pathology and ${ }^{2}$ Ultrasound, The Third Affiliated Hospital of Zhengzhou University, Zhengzhou, Henan 450052, P.R. China; ${ }^{3}$ Division of Transplantation Immunology, National Research Institute for Child Health and Development, Tokyo 157-8535, Japan; ${ }^{4}$ Pathogen Biology Laboratory, The Basic Medical College of Guangdong Medical University, Dongguan, Guangdong 523000, P.R. China
\end{abstract}

Received October 9, 2016; Accepted January 3, 2018

DOI: $10.3892 / \mathrm{ol} .2018 .9327$

\begin{abstract}
MicroRNAs serve a role in the development of ovarian cancer (OC). The present study investigated whether let-7c is able to regulate the proliferation of OC cells by targeting cell division cycle 25A (CDC25a). The reverse transcription-quantitative polymerase chain reaction was performed to detect the expression of let-7c in OC specimens. Let-7c agomir was transfected into OC cells, and the proliferation and apoptosis of OC cells were detected. A dual-luciferase assay and western blotting were performed to analyze whether CDC25a was the target gene of let-7c as well as its interaction site. The results revealed that, in OC tissue, let-7c was downregulated when compared with normal ovarian tissue. A Cell Counting Kit-8 (CCK8) assay, colony formation assay and flow cytometry demonstrated that increased expression of let-7c was able to inhibit the proliferation and increase the apoptosis of OC cells. Western blotting revealed that upregulated let-7c is able to decrease the expression of CDC25a, and a dual-luciferase assay and a recovery assay demonstrated that let-7c was able to regulate the expression of the 3 ' untranslated region of $\mathrm{CDC} 25 \mathrm{a}$. Therefore, the roles of let-7c in inhibiting the proliferation and promoting the apoptosis of OC cells may be realized through the regulation of the expression of CDC25a. The results of the present study revealed that let-7c may be a novel target in the diagnosis and treatment of OC.
\end{abstract}

\section{Introduction}

Ovarian cancer (OC) is a common gynecological malignancy (1), ranking third among the worldwide incidence of gynecological

Correspondence to: Professor Xianxu Zeng, Department of Pathology, The Third Affiliated Hospital of Zhengzhou University, 7 Kangfuqian Street, Zhengzhou, Henan 450052, P.R. China

E-mail: dongmeileicn@163.com

Key words: ovarian cancer, let-7c, cell division cycle 25a, proliferation cancers, with its mortality rate ranking the first (2). Owing to its subtle onset and atypical early-stage clinical symptoms, almost $70 \%$ of OC patients present with stage III or IV OC when diagnosed (3). With continued investigation into OC pathogenesis, the search for early diagnosis and chemotherapy-sensitive markers has gained increased attention.

MicroRNAs (miRNAs/miRs) are a class of endogenous non-coding small RNA molecules which serve roles in cell growth, differentiation, metabolism and the cell cycle $(4,5)$. The mutation, deletion and aberrant expression of miRNAs is associated with the occurrence and development of human tumors. Upregulation of miRNAs which have tumor suppressing roles is anticipated to be a novel approach in cancer gene therapy $(6,7)$.

Let-7 was one of the first miRNAs to be identified in humans, and the $5^{\prime}$ end of human let-7 miRNA family (let-7a-1, let-7a-2, let-7a-3, let-7b, let-7c, let-7d, let-7e, let-7f-1, let-7f-2, let-7g, let-7i, miR-202 and miR-98) contains one highly conserved nucleotide seed sequence which is necessary for miRNA target binding $(8,9)$. Through the regulation of target genes, let-7 is involved in multiple biological processes including cell proliferation, differentiation, apoptosis, hormone secretion, metabolism, immune regulation and tumorigenesis. Downregulated expression of let-7 has been observed in several tumor tissues and cells including breast, lung, prostate and hepatocellular cancer (10-13). Let-7 is abundant in ovaries, and serves an important role in reproductive control during distinct developmental stages, during which the expression levels of let-7 in ovary cells are different (14). A previous study demonstrated that let-7c is involved with various ovarian physiological and pathological processes via regulation of its target genes (15). However, the underlying molecular mechanisms of let-7c in OC are not well understood. Prior investigation has revealed that cell division cycle 25A (CDC25a) is the target gene of let-7c (16). As a cell cycle regulatory protein, the abnormal expression of CDC25a is associated with the occurrence and development of multiple tumors (17-21). In the present study, the biological functions of let-7c in the development and progression of $\mathrm{OC}$ were investigated. The extent by which let-7c exhibits its cancer suppressive roles through the regulation of CDC25a expression levels was also investigated. 


\section{Materials and methods}

Clinical samples. A total of $52 \mathrm{OC}$ tissue samples resected in Henan Provincial Maternal and Child Health Institute (Zhengzhou, China) from March 2014 to March 2015 were selected, including 21 poorly differentiated cases, 18 mid-differentiated cases and 13 well-differentiated cases. The patients were aged between 27 and 68 years, with a mean age of 55.7 years. According to the clinical staging criteria of OC by the International Federation of Gynecology and Obstetrics (FIGO) (22), 8 cases were in stage I, 10 cases were in stage II, 19 cases were in stage III and 15 cases were in stage IV. The tissue samples included 33 cases of serous adenocarcinoma and 19 cases of mucinous adenocarcinoma. No chemotherapy, radiotherapy or immunotherapy was being performed when sampling the specimens, and all samples were confirmed using histology and imaging. In the present study, all patients provided written informed consent, and the Ethics Committee of Third Affiliated Hospital of Zhengzhou University approved the present study.

Cell culture and transfection. OC cell lines SKOV3 and ES2 were purchased from the Type Culture Collection of the Chinese Academy of Sciences (Shanghai, China), cultured in RPMI-1640 cell culture medium containing 10\% fetal bovine serum (FBS; Gibco; Thermo Fisher Scientific, Inc., Waltham, MA, USA) at $37^{\circ} \mathrm{C}$ with $5 \% \mathrm{CO}_{2}$.

During transfection, the OC cells were seeded in 6-well plates at a concentration of $5 \times 10^{4}$ cells/well, and when $80 \%$ of the cells had fused, $50 \mathrm{nM}$ let-7c agomir was transfected into ES2 and SKOV3 cells using a Lipofectamine ${ }^{\mathrm{TM}} 2000$ kit (Invitrogen, Thermo Fisher Scientific, Inc.), according to the manufacturer's protocol. Let-7c agomir (5'-UGAGGUAGU AGGGUUGUAUGGUU-3') and the negative control (5'-CAG UACUUUUGUGUAGUACAA-3') were designed and synthesized by Guangzhou Ribobio Co., Ltd. (Guangzhou, China). The culture medium was changed $6 \mathrm{~h}$ after transfection for a further $24 \mathrm{~h}$ in culture, and the cells were then collected for various assays.

RNA extraction and reverse transcription-quantitative polymerase chain reaction (RT- $q P C R)$. The total RNA in the OC and normal ovarian tissues was extracted using TRIzol reagent (Invitrogen; Thermo Fisher Scientific, Inc.). An RNA extraction kit (Invitrogen; Thermo Fisher Scientific, Inc.) was used according to the manufacturer's protocol to extract the total RNA from the OC cells. A NanoDrop 1000 spectrophotometer (Thermo Fisher Scientific, Inc.) was used to determine the concentration and purity of the extracted RNA, with the optical density $260 / 280 \mathrm{~nm}$ value close to 1.8 , indicating that the purity complied with the test requirements.

RT-qPCR was performed to detect the expression of let-7c in these specimens. An RNA Reverse Transcription kit (Thermo Fisher Scientific, Inc.) was used according to the manufacturer's protocol to reverse-transcribe $1.0 \mu \mathrm{g}$ total RNA into cDNA; ABI Power SYBR-Green PCR Master Mix (Applied Biosystems; Thermo Fisher Scientific, Inc.) qPCR amplification was performed according to the manufacturer's protocol to detect the target fragments. An ABI 7500 Fast Real-Time PCR system (Applied Biosystems; Thermo
Fisher Scientific, Inc.) was used according to the manufacturer's protocol to perform PCR, with a Homo sapiens let-7c specific primer sequence (Applied Biosystems; Thermo Fisher Scientific, Inc.). The copy number of RNU6B was used as the correction base, to detect the concentration of let-7c, which was expressed as a relative expression level using the $2^{-\Delta \Delta C q}$ method (23). The primer sequences used were as follows: miRNA-let-7c: 5'-GCGCGTGAGGTAGTAGGTT-3' (sense) and 5'-GTGCAGGGTCCGAGGT' (anti-sense); U6 5'-GCG CGTCGAAGCGTTC-3' (sense) and 5'-GTGAGGGTCCGA GGT-3' (anti-sense). Hot start PCR conditions were $10 \mathrm{sec}$ at $95^{\circ} \mathrm{C}, 20 \mathrm{sec}$ at $60^{\circ} \mathrm{C}$ and $10 \mathrm{sec}$ at $72^{\circ} \mathrm{C}$ for 40 cycles.

Western blot analysis. The protein samples of each group were collected. The cells were digested with $0.25 \%$ trypsin and the cell stocks were centrifuged at $156 \mathrm{x} \mathrm{g}$ for $5 \mathrm{~min}$ at $4^{\circ} \mathrm{C}$. The cells were resuspended with $1 \mathrm{ml}$ phosphate suffered saline (PBS) and centrifuged at $156 \mathrm{x}$ g for $5 \mathrm{~min}$ at $4^{\circ} \mathrm{C}$. These cells were then lysed with radioimunoprecipitation assay buffer (Beyotime Institute of Biotechnology, Haimen, China) to lyse the cells, and coomassie brilliant blue was used to determine the protein concentration. Following separation using SDS-PAGE $(10 \% \mathrm{gel})$, the required proteins $(50 \mu \mathrm{g})$ were transferred onto a polyvinylidene difluoride film, followed by $1 \mathrm{~h}$ of blocking using 5\% skimmed milk in Tris-buffered saline with Tween-20, and overnight incubation at $4^{\circ} \mathrm{C}$ with agitation with diluted primary antibody (1:500; rabbit anti-human CDC25a antibody; cat. no. SC-97; Santa Cruz Biotechnology, Inc., Dallas, TX, USA). Following washing using Tris-buffered saline containing Tween-20 (TBST) for 4x15 min, diluted secondary antibody (1:1,000; horseradish peroxidase-labeled IgG; cat. no. SC-2357; Santa Cruz Biotechnology, Inc.) was added for $1 \mathrm{~h}$ to incubate, followed by TBST washing for $4 \times 15$ min. An ECL Western Blotting Detection kit was then used to detect the signals (GE Healthcare, Chicago, IL, USA) with GAPDH (1:500; cat. no. sc-47724; Santa Cruz Biotechnology, Inc.) as the reference to calculate the relative expression of the proteins.

Detection of cellular proliferation using Cell Counting Kit-8 (CCK-8). CCK-8 contains WST-8 [chemical name, 2-(2-meth oxy-4-nitrophenyl)-3-(4-nitrophenyl)-5-(2,4-disulfonic acid benzene)-2H-tetrazolium monosodium salt], which is able to be reduced to a highly water-soluble formazan dye by the dehydrogenase in the electron carrier 1-methoxy-5-methylphenazinium dimethyl sulfate in cells. The amount of formazan produced is proportional to the number of viable cells. Therefore, this trait may be utilized directly as a cell proliferation assay. A CCK-8 kit (Dojindo Molecular Technologies, Inc., Kumamoto, Japan) was used to detect the effect of let-7c on cellular proliferation. Cells in the exponential growth phase were seeded in 96-well plates ( $2 \times 10^{3}$ cells/well), with $10 \mu \mathrm{l}$ WST- 8 solution added into each well at $24,48,72$ and $96 \mathrm{~h}$ respectively, according to the manufacturer's protocol to detect the cell proliferation. Results were expressed as the optical density value of each well at $450 \mathrm{~nm}$.

Plate clone assay. Low melting point agarose solution (0.6\%) was mixed with RPMI-1640 medium containing 10\% FBS (1:1), added into 6-well plates, and allowed to cool and solidify at room temperature. The cells of each group in the exponential growth phase following transfection were suspended 
in the aforementioned culture medium, and then added into 6-well plates in which the lower gel layer had already solidified. Following a 12 -day incubation at $37^{\circ} \mathrm{C}$, the cells were stained using $1 \mathrm{ml} 1 \%$ crystal violet to count the cell clones with the naked eye and light microscopy (x100 magnification). The cell clones with more than 50 cells were counted as one monoclone, and the mean number of clones on the plate was used to calculate the cell clone ability.

Detection of apoptosis by flow cytometry. The cells were digested by $0.2 \%$ trypsin at $37^{\circ} \mathrm{C}$ for $2 \mathrm{~min}$ and centrifuged at $156 \mathrm{x} \mathrm{g}$ for $5 \mathrm{~min}$ at $4^{\circ} \mathrm{C}$, and the cells were counted following suspension in PBS $\left(1 \times 10^{6}\right.$ cells/ml $72 \mathrm{~h}$ post-transfection. An annexin V-fluoroscein isothiocyanate (FITC)/propidium iodide (PI) Apoptosis Detection kit (BestBio, Shanghai, China) was used to detect the proportion of cells undergoing apoptosis and flow cytometry (BD Biosciences, Franklin Lakes, NJ, USA) was used to determine the cell apoptosis within $30 \mathrm{~min}$.

Dual-luciferase reporter gene assay. Three target gene prediction databases, TargetScan (http://www.targetscan.org), PicTar (http://pictar.mdc-berlin.de) and miRanda (http://microrna. sanger.ac.uk), were used to predict the target gene of let-7c, with CDC25a identified as the potential target. The DNA of healthy human subjects was extracted using a Human blood genomic DNA extraction kit (Promega Corporation, Madison, WI, USA) following the provision of written informed consent, and the CDC25a 3' untranslated region (UTR) that included the let-7c-binding sites was amplified using PCR. The reaction protocols were as follows: $95^{\circ} \mathrm{C}$ pre-degeneration, cycle $95^{\circ} \mathrm{C}$ for $10 \mathrm{sec}$ degeneration; $1^{\circ} \mathrm{C}$ annealing from $65^{\circ} \mathrm{C}$ each cycle, $72^{\circ} \mathrm{C}$ extension for $2 \mathrm{~min}$, for 10 cycles; $55^{\circ} \mathrm{C}$ annealing, 15 cycles of; $72^{\circ} \mathrm{C}$ for $7 \mathrm{~min}$ and $4^{\circ} \mathrm{C}$ preservation. TaqMan Universal PCR Master Mix (Applied Biosystems). The primer sequences of CDC25A used were as follows: 5'-CCGCTCGAG GCGGCAGGACCAGCCAG-3' (sense) and 5'-GAATGCGGC CGCTCAGAGCTTCCAACAGTTGGTTAG-3' (anti-sense). The amplified CDC25a was then recovered by AxyPrep DNA gel recovery kit (Axygen; Corning Incorporated, Corning, NY, USA) following $1 \%$ agarose electrophoresis, and connected with pmirGLO carrier (pmirGLO-CDC25a-wt, wild-type CDC25a) using T4 DNA ligase (Takara Bio, Inc., Otsu, Japan); mutagenic primers targeting the CDC25a 3'UTR seed region were then designed, and amplified using the overlap method, and inserted into the multi-cloning site of pmirGLO vector (pmirGLO-CDC25a-mut, mutant CDC25a). Lipofectamine 2000 was then used to co-transfect the recombinant vectors and let-7c agomir or negative control into OC cells; $48 \mathrm{~h}$ later, a Dual-Luciferase Reporter assay system (Promega Corporation, Madison, WI, USA) was used to measure the dual-luciferase signals in each group, with the Renilla luciferase signal as the reference; each group was tested in triplicate.

Recovery assay. The CDC25a fragment free of 3'UTR was amplified and inserted into the eukaryotic expression vector pcDNA3.1 (pcDNA3.1-CDC25a). pcDNA3.1-CDC25a was then transfected into SKOV3 cells; meanwhile, let-7c agomir or negative control was also transfected using Lipofectamine ${ }^{\circledR} 2000$ (Invitrogen; Thermo Fisher Scientific, Inc.) according to the manufacturer's protocol. The cells of each group were collected $48 \mathrm{~h}$ later, expression levels of CDC25a protein were investigated using western blotting; and apoptosis was investigated using flow cytometry.

Statistical analysis. SPSS (version 18.0; SPSS, Inc., Chicago, IL, USA) was used for statistical analysis. Data are expressed as the mean \pm standard deviation. The differences between groups was analyzed using Student's t-test or analysis of variance with Student-Newman-Keuls method as a post-hoc test. $\mathrm{P}<0.05$ was considered to indicate a statistically significant difference.

\section{Results}

Expression oflet-7c in OC tissue. The expression levels of let-7c in $52 \mathrm{OC}$ cases determined using RT-qPCR demonstrated that, compared with normal tissue, let-7c was significantly downregulated in the $\mathrm{OC}$ tissue $(\mathrm{P}<0.05$; Fig. $1 \mathrm{~A})$; further analysis of the association of let-7c expression with the pathological factors of patients revealed that let-7c was associated with lymph node metastasis (Fig. 1B) and clinical stage $(\mathrm{P}<0.05$; Fig. 1C and Table I), the expression levels of let-7c in samples from patients with lymph node metastasis were decreased compared with those with no lymph node metastasis, and were decreased in the higher clinical grading group; however, there was no observable association with age, pathological type or differentiation degree $(\mathrm{P}>0.05$; Table I).

Effects of let-7c on the proliferation of OC cells. CCK-8 and plate clone assays were performed to analyze the effects of let-7c on the proliferation of OC cells. A CCK-8 assay revealed that, after $48 \mathrm{~h}$ of transfection of the let-7c agomir into the OC cell lines, the relative proliferation rates of SKOV3 and ES2 were significantly decreased compared with the blank group and the control group $(\mathrm{P}<0.05)$, and no statistically significant difference was identified between the blank group and the control group at any time point $(\mathrm{P}>0.05$; Fig. 2$)$. The plate clone assay demonstrated that the let-7c agomir significantly decreased the number of OC cell colonies $(\mathrm{P}<0.05$; Fig. 3 ), suggesting that the overexpression of let-7c inhibited the proliferative abilities of the OC cell lines SKOV3 and ES2.

Effects of let-7c on apoptosis of OC cells. Flow cytometry revealed that, following transfection with let-7c agomir, the apoptotic rates of SKOV3 and ES2 cells were significantly increased compared with the control group ( $\mathrm{P}<0.05$; Fig. 4$)$, suggesting that the overexpression of let-7c leads to the apoptosis of SKOV3 and ES2 cells.

Expression inhibition of CDC25a mRNA 3'UTR by let-7c. The miRNA bioinformatics database analysis identified that CDC25a was the target gene of let-7c (Fig. 5A). The present study inserted human CDC25a wild-type and mut-type 3'UTRs into the dual-luciferase reporter vector pmirGLO, and co-transfected this recombinant vector and let-7c agomir or negative control into OC cells to detect the fluorescent signal changes in each group. The results demonstrate that following co-transfection with let-7c agomir and pmirGLO-CDC25a-wt, luciferase activity was significantly inhibited $(\mathrm{P}<0.05$; Fig. 5B); however, the co-transfection of let-7c agomir and 
Table I. Association between let-7c expression and clinical pathological factors in OC.

\begin{tabular}{lcc}
\hline Clinicopathological characteristic & $\mathrm{n}$ & let-7c expression \\
\hline Age, years & & \\
$<50$ & 23 & $0.4741 \pm 0.0770$ \\
$\geq 50$ & 29 & $0.5132 \pm 0.0828$ \\
Tissue type & & \\
Serous adenocarcinoma & 33 & $0.4976 \pm 0.0853$ \\
Mucinous adenocarcinoma & 19 & $0.4929 \pm 0.7768$ \\
Differentiation & & \\
High & 21 & $0.4819 \pm 0.0744$ \\
Medium & 18 & $0.4892 \pm 0.0924$ \\
Poor & 13 & $0.5278 \pm 0.0752$ \\
Clinical stage & & \\
I and II & 18 & $0.5333 \pm 0.0831$ \\
III and IV & 34 & $0.4761 \pm 0.0750$ \\
Lymph node metastasis & & \\
No (-) & 24 & $0.5218 \pm 0.0833$ \\
Yes (+) & 28 & $0.4737 \pm 0.0751$ \\
\hline
\end{tabular}

Values are the median \pm standard deviation. ${ }^{\mathrm{a}} \mathrm{P}<0.05$.
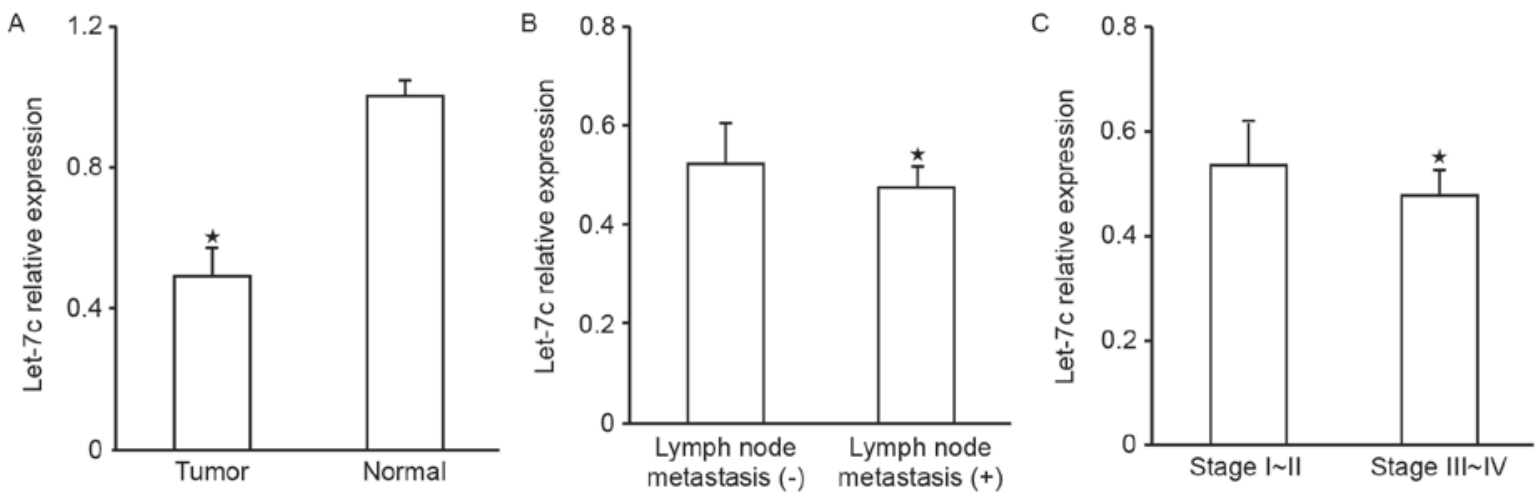

Figure 1. Detection of let-7c expression level in OC tissue using the reverse transcription-quantitative polymerase chain reaction. (A) Let-7c was significantly increased in $\mathrm{OC}$ tissue compared with normal tissue ( $\mathrm{P}<0.05)$. (B) Let-7c expression levels are significantly decreased in metastatic lymph nodes compared with non-metastatic lymph node tissue ( $\mathrm{P}<0.05)$. (C) In OC tissues, the higher the clinical stage, the lower the let-7c expression level (" $\mathrm{P}<0.05)$. OC, ovarian cancer.

pmirGLO-CDC25a-mut demonstrated no significant change in luciferase activity ( $\mathrm{P}>0.05$; Fig. 5B), suggesting that let-7c may combine with the CDC25a 3'UTR seed region, thus resulting in the downregulation of the CDC25a gene. Western blotting results also revealed that let-7c agomir was able to decrease the expression of CDC25a protein in OC cells (Fig. 5C).

Overexpression of CDC25a is able to recover the pro-apoptotic effect of let-7c towards OC cells. In order to further explore the targets of let-7c, pcDNA3.1-CDC25a was constructed and co-transfected alongside let-7c agomir or a negative control into SKOV3 cells; furthermore, let-7c agomir or a negative control were separately transfected into SKOV3 cells. An apoptosis assay demonstrated that when only let-7c agomir was transfected, was the apoptotic rate significantly increased; however, the apoptotic rate was significantly decreased when pcDNA3.1-CDC25a was transfected or pcDNA3.1-CDC25a and let-7c agomir were co-transfected into the cells (Fig. 6A). Western blot analysis revealed that, following the co-transfection of pcDNA3.1-CDC25a and let-7c agomir, CDC25a protein was increased, indicating that pcDNA3.1-CDC25a is able to recover the inhibition of let-7c towards the expression of CDC25a protein $(\mathrm{P}<0.05$; Fig. 6B). This further illustrates that let-7c may act on the CDC25a 3'UTR seed region, thus regulating the CDC25a expression exhibiting pro-apoptotic effects towards OC.

\section{Discussion}

miRNAs are able to regulate transcription by binding to the 3'UTR of target genes, thus serving a role in a variety 

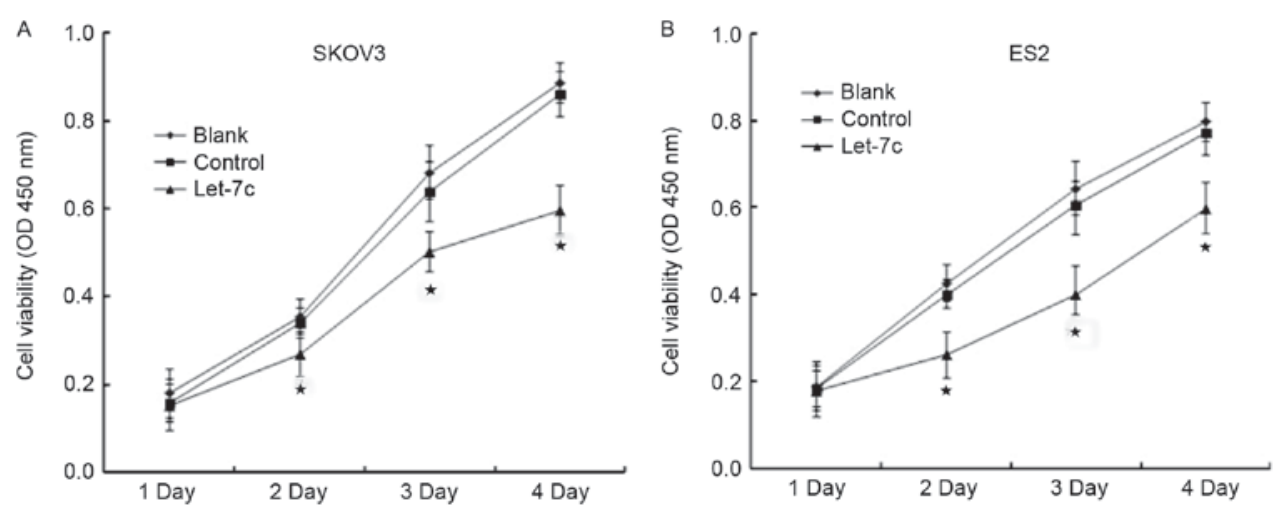

Figure 2. Let-7c agomir significantly inhibits the proliferation of OC cells. A Cell Counting Kit-8 assay revealed that compared with the blank group and the control group, the proliferation abilities of (A) SKOV3 and (B) ES2 were significantly decreased at 2, 3 and 4 days after let-7c agomir transfection compared with the control $(" \mathrm{P}<0.05)$. OD, optical density. OC, ovarian cancer.
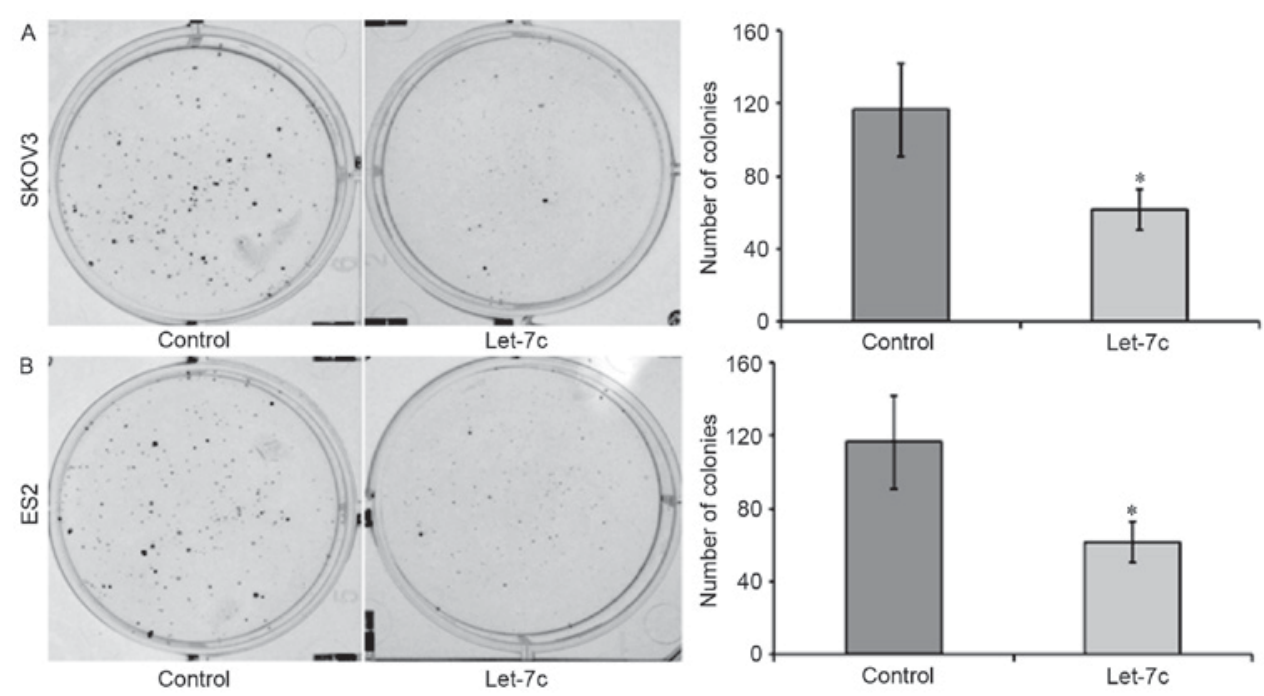

Figure 3. Overexpression of let-7c inhibits the colony formation ability of OC cells. The let-7c agomir-transfected OC cell lines (A) SKOV3 and (B) ES2 exhibited significantly decreased cell colony numbers compared with control cells ( $\mathrm{P}<0.05)$. OC, ovarian cancer.

of physiological processes (24). A previous study revealed that the expression profiles of miRNAs in tumor tissue and normal tissue exhibit significant differences, and the expression levels of miRNAs are associated with the development, differentiation, metastasis and prognosis of tumor cells (25). Previous studies have analyzed miRNA expression profiles in order to investigate miRNAs in a variety of malignant tumors, including OC and OC cell lines, expecting to identify miRNAs with diagnostic potential $(26,27)$. Zhang et al $(28)$ detected the expression of 173 mature miRNAs in OC cell lines and human ovarian surface epithelial cell lines, and identified 35 miRNAs with significant expression differences, among which 31 were downregulated and four were upregulated. Iorio et al (29) analyzed the miRNA expression profiles in OC tissue, and revealed that miRNA was not only differentially expressed between tumor and normal tissues, but also differentially expressed in different histological subtypes.

miRNAs themselves may become the target of cancer therapies which alter the expression of miRNA so as to regulate the target genes and treat cancer. Mature let-7 is able to bind to the $3^{\prime}$ UTR of its target gene and degrade the target mRNA or inhibit its translation. Han et al (30) overexpressed let $-7 \mathrm{c}$, which inhibited the invasion and metastasis of colorectal cancer cells. Zhao et al (31) demonstrated that let-7c acts directly on downstream genes mitogen-activated protein kinase kinase kinase kinase 3 and integrin subunit $\beta 3$, thus inhibiting the invasion and metastasis of non-small cell lung cancer. Wang et al (32) demonstrated that let-7c inhibits the proliferation of lung cancer cells by acting on tribbles homolog 2. Therefore, during the development of OC, the similar role of let-7c as a cancer suppressing gene may also result from its regulation of target genes. The present study detected the let- $7 \mathrm{c}$ expression levels in OC tissue and normal ovarian tissue, and demonstrated that let-7c was downregulated in OC, with its level associated with lymph node metastasis and clinical stage, suggesting that miR-26a has certain associations with the occurrence of OC. At the same time, following transfection of let-7c agomir into SKOV3 and ES2 cells, it was revealed that the OC cell proliferation was decreased; however, the apoptotic rate was increased, suggesting that let-7ca is able to inhibit the proliferation of OC, consistent with its biological effects in other tumors.

Bioinformatics analysis revealed that $\mathrm{CDC} 25$ a may be a potential target site of let-7c. The CDC25 gene can express 

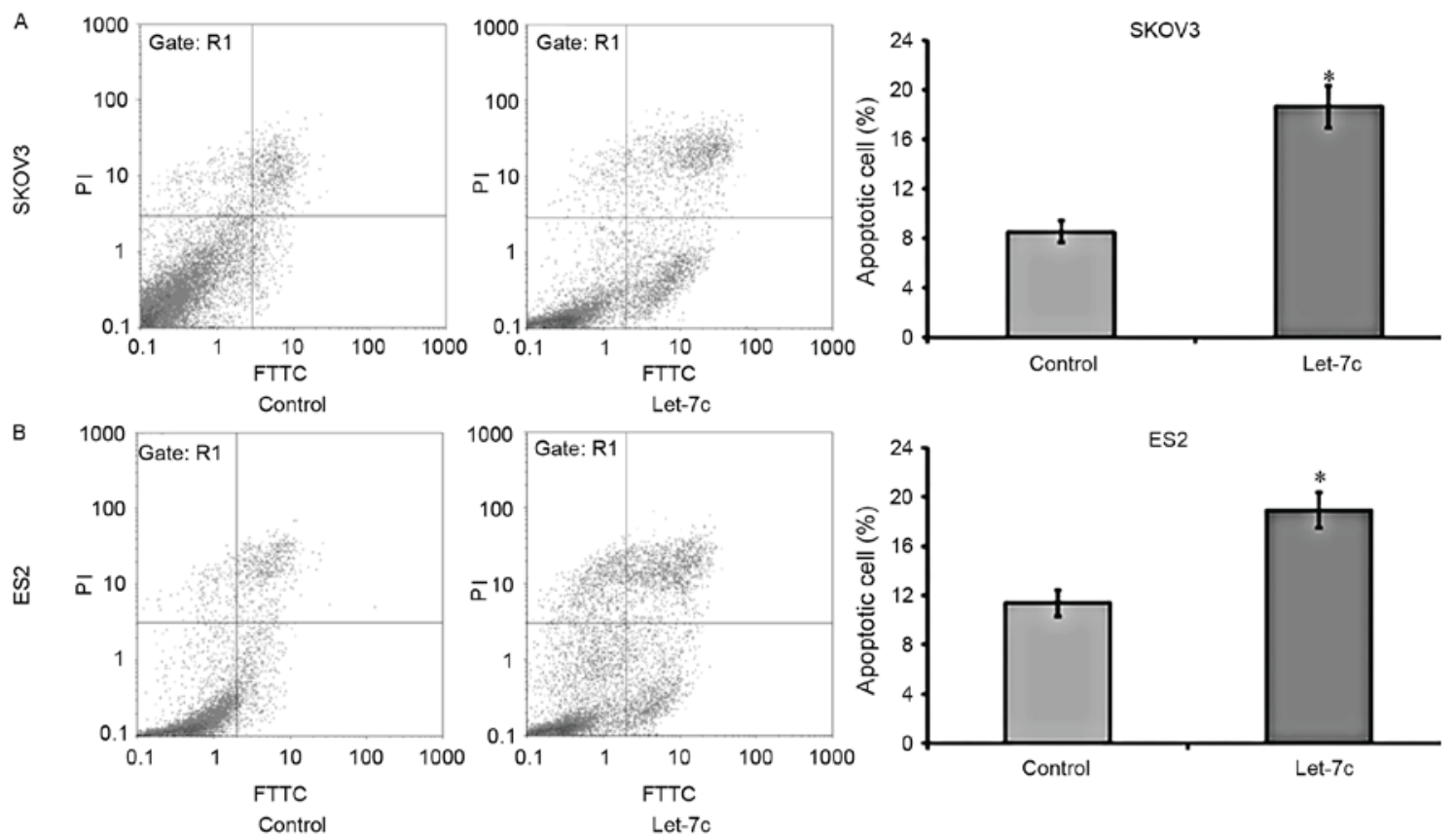

Figure 4. Overexpression of let-7c may lead to the apoptosis of SKOV3 and ES2 cells. The apoptotic rates of (A) SKOV3 and (B) ES2 cells were significantly increased when compared with the control group following transfection with let-7c agomir ( $\mathrm{P}<0.05)$. PI, propidium iodide.

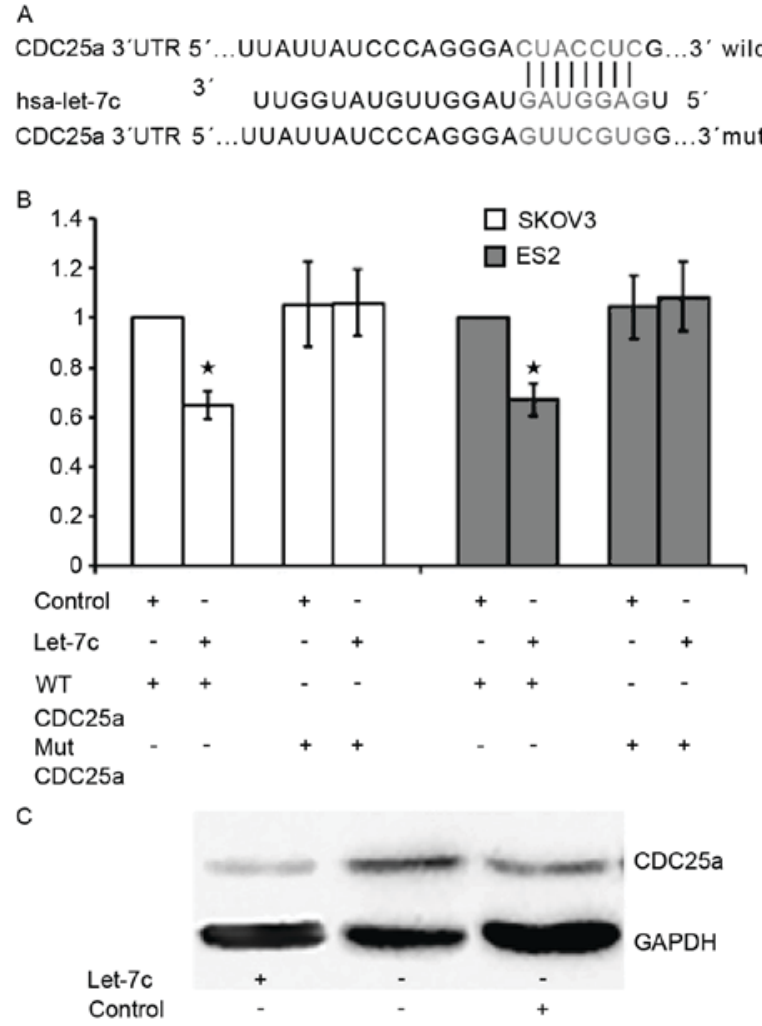

Figure 5. Let-7c acts on CDC25a 3'UTR and regulates its expression. (A) Let-7c acts on CDC25a 3'UTR seed region sequence. (B) A dual-luciferase reporter gene assay demonstrated that the co-transfection of let-7c agomir and pmirGLO-CDC25a-wt significantly decreases the luciferase activity of SKOV3 cells when compared with the control group (miR-NC and pmirGLO-CDC25a-wt co-transfection group; ${ }^{*} \mathrm{P}<0.05$ ); the co-transfection of let-7c agomir and pmirGLO-CDC25a-mut does not significantly alter the luciferase activity in comparison with the control group (negative control and pmirGLO-CDC25a-mut co-transfection group) ("P>0.05). (C) Western blot analysis revealed that the overexpression of let-7c suppresses the CDC25a protein level in SKOV3 cells. CDC25a, cell division cycle 25a; Hsa, Homo sapiens; WT, wild-type; Mut, mutant.

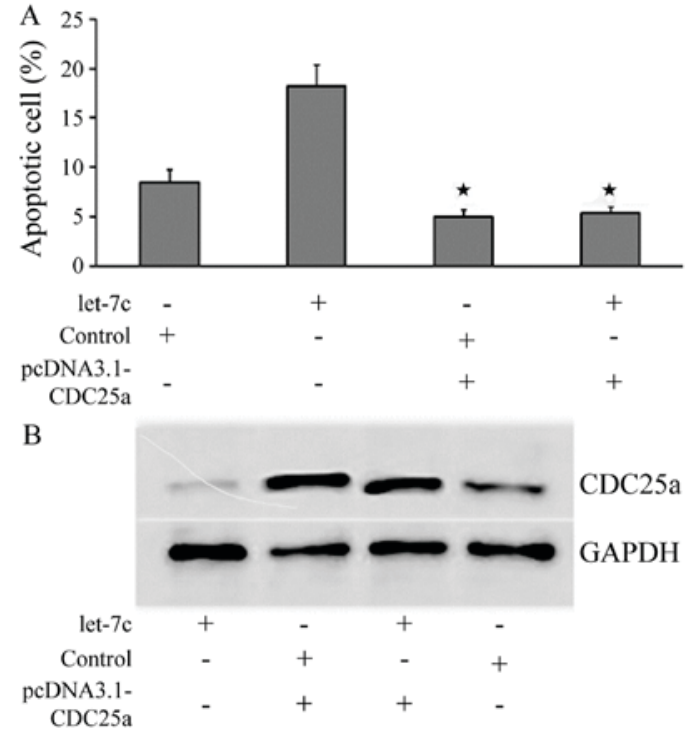

Figure 6. Overexpression of CDC25a may recover the pro-apoptotic effects of let-7c towards OC cells. (A) The co-transfection of CDC25a 3'UTR-free pcDNA3.1-CDC25a and let-7c agomir into SKOV3 cells significantly decreased the apoptotic rate of the cells $\left({ }^{*} \mathrm{P}<0.05\right)$. (B) Western blot analysis revealed that CDC25a 3'UTR-free pcDNA3.1-CDC25a is able to recover the let-7c agomir-inhibited expression of $\mathrm{CDC} 25 \mathrm{a}$ protein in SKOV3 cells, thus resulting in the upregulation of CDC25a protein. CDC25a, cell division cycle 25a.

CDC25 phosphatase protein, a cell cycle regulatory protein which serves a role in the normal cell cycle (33). In a series of human malignancies including hepatocellular carcinoma, ovarian cancer, colorectal cancer, esophageal cancer and non-Hodgkin's lymphoma, CDC25a exhibits increased expression levels (17-21). CDC25a exhibits pro-cancer traits in two distinct manners. First, it is able to promote cell proliferation (34). When cells enter $\mathrm{S}$ phase, CDC25a activates the cyclin 
E-cyclin dependent kinase-2 (CDK2) and cyclin A-CDK2 complexes by dephosphorylation, thus promoting cells to enter $\mathrm{S}$ phase (35). If the CDC25a protein is overexpressed, it may cause cells to rapidly enter $\mathrm{S}$ phase from $\mathrm{G}$ phase, followed by an increase in DNA synthesis, malignant cell proliferation or even development of cancer (36). Secondly, CCD25a is considered to be a checkpoint gene, and acts simultaneously on two checkpoints, the $\mathrm{G}_{1} / \mathrm{S}$ phase and the $\mathrm{G}_{2} / \mathrm{M}$ phase, thus serving roles in DNA damage and repair (37). Therefore, the overexpression of CDC25a may lead to disorders of cell cycle regulation and decreased response to DNA damage, so abnormal cell proliferation or even tumors may be observed as a result of CDC25a overexpression.

Western blotting revealed that the overexpression of let-7c may lead to the downregulation of CDC25a protein in OC cells. CDC25a 3'UTR was then cloned into the dual-luciferase reporter vector pmirGLO, and the results revealed that let-7c is able to bind to the CDC25a 3'UTR seed region, thus negatively regulating its expression. The overexpression of CDC25a revealed by the recovery assay may restore the pro-apoptotic effects of let-7c towards OC cells. This further demonstrates that let-7c may bind to CDC25a mRNA 3'UTR, thus regulating its expression, and inhibiting the proliferation and inducing the apoptosis of OC cells. Therefore, CDC25a may possibly act as a target gene of let-7c, thus regulating the malignant proliferation of OC cells.

The development of OC is regulated by a complex network of numerous cytokines, enzymes and genes, among which let- $7 \mathrm{c}$ serves a role as a tumor suppressor, thus affecting the development, as well as staging and treatment, of tumors. Previous research has also demonstrated that let-7c may bind to several oncoproteins and several key regulatory factors involved in the mitotic pathway and regulate their expression (38). CDC25a is only one target gene of let-7c $(16,39)$. The activities of CDC25a are regulated by multiple mechanisms, including the ubiquitin ligase anaphase-promoting complex/cyclosome (40) and transforming growth factor $\beta$ (41). Therefore, the present study demonstrated only that let-7c may target CDC25a, thus inhibiting the proliferation of OC cells; further research into other biological functions of let-7c is required.

The abnormal expression of let-7c in OC tissue is associated with the proliferation of OC; let-7c may act on CDC25a 3'UTR and inhibit its expression, thus serving a similar role to a tumor suppressor gene. Further in-depth studies into the target genes and mechanisms of let-7c may reveal its potential as a novel diagnostic and therapeutic target in OC.

\section{Acknowledgements}

Not applicable.

\section{Funding}

No funding was received.

\section{Availability of data and materials}

All data generated or analyzed during this study are included in this published article.

\section{Authors' contributions}

XZ designed the research. WG, WZ, QZ, ZB and JC conducted the experiments. TJ TC, DL and CL analyzed the data. The manuscript was drafted by WG and WZ. All authors read and approved the final manuscript.

\section{Ethics approval and consent to participate}

This study was approved by the Ethics Committee of Third Affiliated Hospital of Zhengzhou University and written informed consent was obtained from all participants.

\section{Patient consent for publication}

All participants provided written informed consent for publication.

\section{Competing interests}

The authors declare that they have no competing interests.

\section{References}

1. Siegel RL, Miller KD and Jemal A: Cancer statistics, 2015. CA Cancer J Clin 65: 5-29, 2015.

2. Torre LA, Bray F, Siegel RL, Ferlay J, Lortet-Tieulent J and Jemal A: Global cancer statistics, 2012. CA Cancer J Clin 65: 87-108, 2015.

3. Salani R and Bristow RE: Surgical management of epithelial ovarian cancer. Clin Obstet Gynecol 55: 75-95, 2012.

4. Sokol NS: Small temporal RNAs in animal development. Curr Opin Genet Dev 22: 368-373, 2012.

5. Contreras J and Rao DS: MicroRNAs in inflammation and immune responses. Leukemia 26: 404-413, 2012.

6. Di Leva G, Garofalo M and Croce CM: MicroRNAs in cancer. Annu Rev Pathol 9: 287-314, 2014.

7. Ruan K, Fang X and Ouyang G: MicroRNAs: Novel regulators in the hallmarks of human cancer. Cancer Lett 285: 116-126, 2009.

8. Reinhart BJ, Slack FJ, Basson M, Pasquinelli AE, Bettinger JC, Rougvie AE, Horvitz HR and Ruvkun G: The 21-nucleotide let-7 RNA regulates developmental timing in caenorhabditis elegans. Nature 403: 901-906, 2000.

9. Roush S and Slack FJ: The let-7 family of microRNAs. Trends Cell Biol 18: 505-516, 2008.

10. Yan Y, Zhang F, Fan Q, Li X and Zhou K: Breast cancer-specific TRAIL expression mediated by miRNA response elements of let-7 and miR-122. Neoplasma 61: 672-679, 2014.

11. Tsai CH, Lin LT, Wang CY, Chiu YW, Chou YT, Chiu SJ, Wang HE, Liu RS, Wu CY, Chan PC, et al: Over-expression of cofilin-1 suppressed growth and invasion of cancer cells is associated with up-regulation of let-7 microRNA. Biochim Biophys Acta 1852: 851-861, 2015.

12. Liu C, Kelnar K, Vlassov AV, Brown D, Wang J and Tang DG: Distinct microRNA expression profiles in prostate cancer stem/progenitor cells and tumor-suppressive functions of let-7. Cancer Res 72: 3393-3404, 2012.

13. Xie K, Liu J, Zhu L, Liu Y, Pan Y, Wen J, Ma H, Zhai X and $\mathrm{Hu} \mathrm{Z}$ : A potentially functional polymorphism in the promoter region of let-7 family is associated with survival of hepatocellular carcinoma. Cancer Epidemiol 37: 998-1002, 2013.

14. Kang L, Cui X, Zhang Y, Yang C and Jiang Y: Identification of miRNAs associated with sexual maturity in chicken ovary by Illumina small RNA deep sequencing. BMC Genomics 14: 352, 2013.

15. Helland Å, Anglesio MS, George J, Cowin PA, Johnstone CN, House CM, Sheppard KE, Etemadmoghadam D, Melnyk N, Rustgi AK, et al: Deregulation of MYCN, LIN28B and LET7 in a molecular subtype of aggressive high-grade serous ovarian cancers. PLoS One 6: e18064, 2011. 
16. Zhu X, Wu L, Yao J, Jiang $\mathrm{H}$, Wang Q, Yang Z and Wu F: MicroRNA let-7c inhibits cell proliferation and induces cell cycle arrest by targeting CDC25A in human hepatocellular carcinoma. PLoS One 10: e0124266, 2015.

17. Broggini M, Buraggi G, Brenna A, Riva L, Codegoni AM, Torri V Lissoni AA, Mangioni C and D'Incalci M: Cell cycle-related phosphatases CDC25A and B expression correlates with survival in ovarian cancer patients. Anticancer Res 20: 4835-4840, 2000.

18. Rodrigues S, Rodrigue CM, Attoub S, Fléjou JF, Bruyneel E, Bracke M, Emami $S$ and Gespach $C$ : Induction of the adenoma-carcinoma progression and Cdc25A-B phosphatases by the trefoil factor TFF1 in human colon epithelial cells. Oncogene 25: 6628-6636, 2006.

19. Nishioka K, Doki Y, Shiozaki H, Yamamoto H, Tamura S, Yasuda T, Fujiwara Y, Yano M, Miyata H, Kishi K, et al: Clinical significance of CDC25A and CDC25B expression in squamous cell carcinomas of the oesophagus. Br J Cancer 85: 412-421, 2001.

20. Aref S, Fouda M, El-Dosoky E, Menessy A, Mabed M, Saleeb M and Zalata K: c-Myc oncogene and Cdc25A cell activating phosphatase expression in non-Hodgkin's lymphoma. Hematology 8: 183-190, 2003.

21. Molinari M, Mercurio C, Dominguez J, Goubin F and Draetta GF: Human Cdc25A inactivation in response to $S$ phase inhibition and its role in preventing premature mitosis. EMBO Rep 1: 71-79, 2000.

22. Pecorelli S: Revised FIGO staging for carcinoma of the vulva, cervix, and endometrium. Int J Gynaecol Obstet 105: 103-104, 2009.

23. Livak KJ and Schmittgen TD: Analysis of relative gene expression data using real-time quantitative PCR and the 2(-Delta Delta C(T)) method. Methods 25: 402-408, 2001.

24. Lewis BP, Shih IH, Jones-Rhoades MW, Bartel DP and Burge CB: Prediction of mammalian microRNA targets. Cell 115: 787-798, 2003.

25. Resnick KE, Alder H, Hagan JP, Richardson DL, Croce CM and Cohn DE: The detection of differentially expressed microRNAs from the serum of ovarian cancer patients using a novel real-time PCR platform. Gynecol Oncol 112: 55-59, 2009.

26. Katz B, Tropé CG, Reich R and Davidson B: MicroRNAs in ovarian cancer. Hum Pathol 46: 1245-1256, 2015.

27. Langhe R: microRNA and ovarian cancer. Adv Exp Med Biol 889: 119-151,2015.

28. Zhang L, Volinia S, Bonome T, Calin GA, Greshock J, Yang N, Liu CG, Giannakakis A. Alexiou P, Hasegawa K, et al: Genomic and epigenetic alterations deregulate microRNA expression in human epithelial ovarian cancer. Proc Natl Acad Sci USA 105 7004-7009, 2008.
29. Iorio MV, Visone R, Di Leva G, Donati V, Petrocca F, Casalini P, Taccioli C, Volinia S, Liu CG, Alder H, et al: MicroRNA signatures in human ovarian cancer. Cancer Res 67: 8699-8707, 2007.

30. Han HB, Gu J, Zuo HJ, Chen ZG, Zhao W, Li M, Ji DB, Lu YY and Zhang ZQ: Let-7c functions as a metastasis suppressor by targeting MMP11 and PBX3 in colorectal cancer. J Pathol 226: $544-555,2012$

31. Zhao B, Han H, Chen J, Zhang Z, Li S, Fang F, Zheng Q, Ma Y, Zhang J, Wu N and Yang Y: MicroRNA let-7c inhibits migration and invasion of human non-small cell lung cancer by targeting ITGB3 and MAP4K3. Cancer Lett 342: 43-51, 2014.

32. Wang PY, Sun YX, Zhang S, Pang M, Zhang HH, Gao SY, Zhang C, Lv CJ and Xie SY: Let-7c inhibits A549 cell proliferation through oncogenic TRIB2 related factors. FEBS Lett 587: 2675-2681, 2013.

33. Kristjánsdóttir K and Rudolph J: Cdc25 phosphatases and cancer. Chem Biol 11: 1043-1051, 2004.

34. Xu X, Yamamoto H, Sakon M, Yasui M, Ngan CY, Fukunaga H, Morita T, Ogawa M, Nagano H, Nakamori S, et al: Overexpression of CDC25Aphosphatase is associated with hypergrowth activity and poor prognosis of human hepatocellular carcinomas. Clin Cancer Res 9: 1764-1772, 2003.

35. Blomberg I and Hoffmann I: Ectopic expression of Cdc25A accelerates the $\mathrm{G}(1) / \mathrm{S}$ transition and leads to premature activation of cyclin E- and cyclin A-dependent kinases. Mol Cell Biol 19: 6183-6194, 1999.

36. Xiao Z, Chen Z, Gunasekera AH, Sowin TJ, Rosenberg SH, Fesik $\mathrm{S}$ and Zhang H: Chk1 mediates $\mathrm{S}$ and $\mathrm{G} 2$ arrests through Cdc25A degradation in response to DNA-damaging agents. J Biol Chem 278: 21767-21773, 2003.

37. Ray D and Kiyokawa $\mathrm{H}$ : CDC25A levels determine the balance of proliferation and checkpoint response. Cell Cycle 6: 3039-3042, 2007.

38. Guo R, Abdelmohsen K, Morin PJ and Gorospe M: Novel MicroRNA reporter uncovers repression of Let-7 by GSK-3 $\beta$. PLoS One 8: e66330, 2013.

39. Zhan M, Qu Q, Wang G, Liu YZ, Tan SL, Lou XY, Yu J and Zhou HH: Let-7c inhibits NSCLC cell proliferation by targeting HOXA1. Asian Pac J Cancer Prev 14: 387-392, 2013.

40. Donzelli M, Squatrito M, Ganoth D, Hershko A, Pagano M and Draetta GF: Dual mode of degradation of Cdc25 A phosphatase. EMBO J 21: 4875-4884, 2002.

41. Ray D, Terao Y, Nimbalkar D, Chu LH, Donzelli M, Tsutsui T, Zou X, Ghosh AK, Varga J, Draetta GF and Kiyokawa H: Transforming growth factor beta facilitates beta-TrCP-mediated degradation of Cdc25A in a Smad3-dependent manner. Mol Cell Biol 25: 3338-3347, 2005. 